\title{
Sublingual and Nasal Transmucosal Drug Delivery for Breakthrough Pain: A Frontier in Cancer Therapy
}

\section{Abeer Al-Ghananaeem*}

Research and Graduate Program, Sullivan University College of Pharmacy, 2100 Gardiner Lane West Campus, Louisville, KY 40205, USA

\section{Editorial}

Breakthrough pain (BTP) is an unpredictable transitory provocation of pain experienced for a short period of time by patients who have chronic pain problem [1]. BTP is highly prevalent in certain patient populations, occurring in $40-80 \%$ of patients with advanced cancer [2] and around $70 \%$ of patients with chronic noncancer pain [3]. A typical BTP episode could reach its peak intensity in three to five minutes and usually last about 30 minutes in total [2]. BTP is frequently detrimental to quality of life. In a study it was reported that $93 \%$ of patients with BTP had a substantial effect on their general activity level and ability to work, and $86 \%$ of patients stated that it affected their enjoyment of life [4].

BTP reduces patients' quality of life, and increases health care costs. It was reported that annual costs of cancer pain-related treatments' health care visits were five times greater for patients with BTP [5]. Thus, it is crucial to provide fast and effective relief of BTP. Rescue doses of short-acting immediate-release oral formulations of opioids such as morphine, oxycodone, hydromorphone, buprenorphine, and methadone are the usual therapy approach for BTP management. However, the absorption of an oral tablet is usually too slowly to treat a BTP typical episode. For example, oral tablets of morphine, with an onset of action of 20 - 30 minutes [6], cannot be fast enough to provide treatment for BTP episode. For that reason, pain management therapy that could be matching the fast onset of BTP continues to be a challenge. Parenteral route for drug administration could provide such fast relief from BTP, but this route usually has low patients' compliance and it is not particularly convenient for patients.

Generally, transmucosal drug delivery through intranasal or sublingual mucosa has been an area of great interest for systemic and central drug delivery resulting in fast onset of action [7-10]. It avoids the hepatic first-pass clearance and has a rapid onset of drug action. Note that the route of administration is an important factor to consider in treatment of fast-onset condition such as BTP. It was reported that patients who administered short-acting opioid had an average time to pain relief as 31 (range 5-75) minutes. No difference was found between oral short-acting opioids, while oral transmucosal fentanyl citrate worked faster than oral morphine, oxycodone, hydromorphone, and methadone [11].

Transmucosal drug delivery of lipophilic opioids has received a great attention due to their quick absorption and fast onset of pain management. Fentanyl is a pure $\mu$ opioid receptor agonist, and is approximately 100 -fold more potent than morphine [12]. Transmucosal administration of lipophilic opioids such as fentanyl revealed a great improvement in BTP management through the fastonset of action reported.

Currently, for the treatment of BTP, fentanyl is marketed in the form of an injectable dosage form, which has always suffered from issues of patient compliance. Fentanyl citrate has also been developed and marketed as a solid intra-oral formulation in the shape of a lollipop. This formulation was intended for oral transmucosal administration in children, to permit fast absorption of fentanyl from the oral cavity. However, it was found that a large percentage of the drug is swallowed from the lollipop [13]. Taking into consideration the inadequacies of the approaches currently available for fentanyl administration, it was shown in rabbits that a sublingual spray provides a quick and reproducible onset of action that may be expedient for use by patients [14]. This dosage form, being a homogeneous solution, allows for rapid absorption of the active ingredient, so as to avoid the potential intraand inter-individual inconsistency resulting from swallowing an oral dosage form.

Furthermore, it was reported that fentanyl administered nasally could work faster than oral transmucosal fentanyl [15]. The advantages of intranasal fentanyl are similar to the sublingual route of administration namely; its bypass of the hepatic first-pass metabolism, high bioavailability, and high patient acceptability. The nasally administered fentanyl may also be a good option for BTP patients suffering from dry mouth. Furthermore, Tetrahydrocannabenol (THC) intranasal delivery, also showed acceptable bioavailability with a short $\mathrm{T}_{\text {max }}$, but the potential irritation to nasal mucous membranes requires further investigation [16].

With the increased demand on enhancing the quality of cancer patients' lives, transmucosal delivery offers compelling opportunities to bring new safe and effective drugs to the market. It offers easy and noninvasive drug delivery methods for BTP treatment in cancer patients due to the resulting quick and short drug onset of action. Extensive research over the last decade provided ample proof of the potential benefits of transmucosal drug delivery in BTP management due to the potential fast onset of drug action, despite the concerns of possible abuse.

With the increase demand on enhancing the quality of cancer patients' life, transmucosal delivery offers compelling opportunities to bring new safe and effective drugs to the market. However, there are many logistics that need to be addressed during drug registration process with the FDA to in order to ensure an effective and safe transmucosal delivery device or system for BTP treatment.

*Corresponding author: Abeer Al-Ghananaeem, Research and Graduate Program, Sullivan University College of Pharmacy, 2100 Gardiner Lane West Campus, Louisville, KY 40205, USA, Tel: 001-502-413-8957; E-mail: aalghananeem@sullivan.edu

Received March 27, 2013; Accepted March 29, 2013; Published April 05, 2013

Citation: Al-Ghananaeem A (2013) Sublingual and Nasal Transmucosal Drug Delivery for Breakthrough Pain: A Frontier in Cancer Therapy. J Bioequiv Availab 5: e29. doi:10.4172/jbb.10000e29

Copyright: (c) 2013 Al-Ghananaeem A. This is an open-access article distributed under the terms of the Creative Commons Attribution License, which permits unrestricted use, distribution, and reproduction in any medium, provided the original author and source are credited. 
Citation: Al-Ghananaeem A (2013) Sublingual and Nasal Transmucosal Drug Delivery for Breakthrough Pain: A Frontier in Cancer Therapy. J Bioequiv Availab 5: e29. doi:10.4172/jbb.10000e29

Page 2 of 2

\section{References}

1. Portenoy RK, Hagen NA (1990) Breakthrough pain: definition, prevalence and characteristics. Pain 41: 273-281.

2. Laverty D (2007) Treating cancer-related breakthrough pain: the oral transmucosal route. Int J Palliat Nurs 13: 326-331.

3. Caraceni A, Martini C, Zecca E, Portenoy RK, Ashby MA, et al. (2004) Breakthrough pain characteristics and syndromes in patients with cancer pain. An international survey. Palliat Med 18: 177-183.

4. Taylor DR, Webster LR, Chun SY, Reinking J, Stegman M, et al. (2007) Impact of breakthrough pain on quality of life in patients with chronic, noncancer pain: patient perceptions and effect of treatment with oral transmucosal fentanyl citrate (OTFC, ACTIQ). Pain Med 8: 281-288.

5. Payne R (2007) Recognition and diagnosis of breakthrough pain. Pain Med 8 Suppl 1: S3-7.

6. Collins SL, Faura CC, Moore RA, McQuay HJ (1998) Peak plasma concentrations after oral morphine: a systematic review. J Pain Symptom Manage 16: 388-402.

7. Al-Ghananeem AM, Traboulsi AA, Dittert LW, Hussain AA (2002) Targeted brain delivery of 17 beta-estradiol via nasally administered water soluble prodrugs. AAPS PharmSciTech 3: E5.

8. Illum L (2003) Nasal drug delivery--possibilities, problems and solutions. J Control Release 87: 187-198.
9. Al-Ghananeem AM, Malkawi AH, Crooks PA (2007) Scopolamine sublingua spray: an alternative route of delivery for the treatment of motion sickness. Drug Dev Ind Pharm 33: 577-582.

10. Hussain AA (1998) Intranasal drug delivery. Adv Drug Deliv Rev 29: 39-49.

11. Zeppetella G (2008) Opioids for cancer breakthrough pain: a pilot study reporting patient assessment of time to meaningful pain relief. J Pain Symptom Manage 35: 563-567.

12. Prommer E (2009) The role of fentanyl in cancer-related pain. J Palliat Med 12: $947-954$.

13. Mystakidou K, Katsouda E, Tsilika E, Parpa E, Vlahos L (2004) Transdermal therapeutic fentanyl-system (TTS-F). In Vivo 18: 633-642.

14. Malkawi AH, Al-Ghananeem AM, Crooks PA (2008) Development of a GC-MS assay for the determination of fentanyl pharmacokinetics in rabbit plasma after sublingual spray delivery. AAPS J 10: 261-267.

15. Vissers D, Stam W, Nolte T, Lenre M, Jansen J (2010) Efficacy of intranasa fentanyl spray versus other opioids for breakthrough pain in cancer. Curr Med Res Opin 26: 1037-1045.

16. Al-Ghananeem AM, Malkawi AH, Crooks PA (2011) Bioavailability of $\Delta^{9}$ tetrahydrocannabinol following intranasal administration of a mucoadhesive ge spray delivery system in conscious rabbits. Drug Dev Ind Pharm 37: 329-334. 